\title{
General Spherically Symmetric Non Singular Black Hole Solutions in Teleparallel Theory of Gravitation
}

\author{
Gamal G.L. Nashed \\ Mathematics Department, Faculty of Science, Ain Shams University, Cairo, Egypt \\ e-mail:nashed@asunet.shams.edu.eg
}

We find the most general spherically symmetric non singular black hole solution in a 'special class of teleparallel theory of gravitation. If $r$ is large enough, the general solution ' coincides with the Schwarzschild solution. Whereas, if $r$ is small, the general solution behaves in a manner similar to that of de Sitter solution. Otherwise it describes a spherically symmetric black hole singularity free everywhere. Moreover, the energy associated with the general solution is calculated using the superpotential given by Møller 1978. 


\section{Introduction}

Møller modified general relativity by constructing a new field theory in teleparallel space [1]. The aim of this theory was to overcome the problem of energy-momentum complex that appears in the Riemannian space [2]. The field equations in this new theory were derived from a Lagrangian which is not invariant under local tetrad rotation. Sáez [3] generalized Møller theory into a scalar tetrad theory of gravitation. Meyer [4] showed that Møller theory is a special case of Poincaré gauge theory [0], 6].

Mikhail et al. [7] obtained two spherically symmetric solutions in Møller's theory when the stress-energy momentum tensor vanishing. They calculated the energy associated with those solutions and found that in one solution the energy does not coincide with the gravitational mass. Shirafuji et al. [8] extended the calculation to all the stationary asymptotic flat space solutions with spherical symmetry, dividing them into two classes, the first in which the components $\left(e_{a 0}\right)$ and $\left(e_{0 \alpha}\right)$ of the parallel vector fields $\left(e_{i \mu}\right)$ tend to zero faster than $1 / \sqrt{r}$ for large $r$ and the second, in which these components go to zero as $1 / \sqrt{r}$. It was found that the equality of the gravitational and inertial masses holds only in the first class. Mikhail et al. [9] also obtained a spherically symmetric solution of Møller's theory starting from a tetrad which contains three unknown functions following Mazumder and Ray [10]. The solution contains one arbitrary function of the radial coordinates $r$, and all the previous solutions can be obtained from it.

Dymnikova 11] derived a static spherically symmetric nonsingular black hole solution in orthodox general relativity assuming a specific form of the stress-energy momentum tensor. This solution practically coincides with the Schwarzschild solution for large $r$, for small $r$ it behaves like the de Sitter solution and describes a spherically symmetric black hole singularity free everywhere [11]. It has been proved that it is possible to treat this specific form of the stress-energy momentum tensor as corresponding to an r-dependent cosmological term $\Lambda_{\mu \nu}$, varying from $\Lambda_{\mu \nu}=\Lambda g_{\mu \nu}$ as $r \rightarrow 0$ to $\Lambda_{\mu \nu}=0$ as $r \rightarrow \infty$ [12]. More recently [13], the spherically symmetric nonsingular black hole has been used to prove that a baby universe inside a $\Lambda$ black hole can be obtained in the case of an eternal black hole. Also it has been shown that the probability of a quantum birth of a baby universe can not be neglected due to the existence of an infinite number of $\Lambda$ white hole structures. Assuming the specific form of the vacuum stress-energy momentum tensor [11, using a spherically symmetric tetrad contains three unknown functions of radial coordinate $r$ only, which was constructed by Robertson [14], the author [15] obtained a general solution of this tetrad when the stress-energy momentum tensor is not vanishing. This solution contains one arbitrary function of the radial coordinates $r$, and all the previous solutions could be derived from it.

The general form of the tetrad, $e_{i}{ }^{\mu}$, having spherical symmetry was given by Robertson [14]. In the Cartesian form it can be written as

$$
\begin{aligned}
& e_{0}{ }^{0}=i A, \quad e_{a}{ }^{0}=C x^{a}, \quad e_{0}{ }^{\alpha}=i D x^{\alpha} \\
& e_{a}{ }^{\alpha}=\delta_{a}^{\alpha} B+F x^{a} x^{\alpha}+\epsilon_{a \alpha \beta} S x^{\beta},
\end{aligned}
$$

where $A, C, D, B, F$, and $S$ are functions of $t$ and $r=\left(x^{\alpha} x^{\alpha}\right)^{1 / 2}$, and the zero ${ }^{t h}$ vector $e_{0}{ }^{\mu}$ has the factor $i=\sqrt{-1}$ to preserve Lorentz signature. We consider an asymptotically flat

${ }^{*}$ In this paper Latin indices $(i, j, \ldots)$ represent the vector number, and Greek indices $(\mu, \nu, \ldots)$ represent the vector components. All indices run from 0 to 3 . The spatial part of Latin indices are denoted by $(a, b, \ldots)$, while that of Greek indices by $(\alpha, \beta, \ldots)$. In the present convention, Latin indices are never raised. 
space-time in this paper, and impose the boundary condition that for $r \rightarrow \infty$ the tetrad (1) approaches the tetrad of Minkowski space-time, $\left(e_{i}{ }^{\mu}\right)=\operatorname{diag}\left(i, \delta_{a}{ }^{\alpha}\right)$.

The aim of the present work is to find a general solution with spherical symmetry in Møller's tetrad theory of gravitation when the vacuum stress-energy momentum tensor is not vanishing and has the same form given by Dymnikova [11]. Moreover, the energy of that solution is calculated. In section 2 we briefly review Møller's tetrad theory of gravitation. In section 3, we study the general solution without the $S$-term (see 1 ), where the remaining unknown functions are allowed to depend on $t$ and $r$ and the general solution with an arbitrary function of $t$ and $r$ is obtained. Then we compare this general solution with that obtained before [15], it is found that this general tetrad is just the $t$-independent case of our general tetrad without the $S$-term. Also we first study the general, spherically symmetric solution with a non-vanishing $S$-term and a solution with one parameter is obtained. In section 4 we calculate the energy content of those general solutions using the superpotential given by Møller in 1978. The final section is devoted to discussion and conclusion.

Computer algebra system Maple 6 is used in some calculations.

\section{Møller's tetrad theory of gravitation}

In a space-time with absolute parallelism the parallel vector fields $e_{i}{ }^{\mu}$ define the nonsymmetric connection

$$
\Gamma_{\mu \nu}^{\lambda} \stackrel{\text { def. }}{=} e_{i}^{\lambda} e_{i \mu, \nu}
$$

where $e_{i \mu, \nu}=\partial_{\nu} e_{i \mu}$. The curvature tensor defined by $\Gamma_{\mu \nu}^{\lambda}$ is identically vanishing, however.

Møller's constructed a gravitational theory based on this space-time. In this theory the field variables are the 16 tetrad components $e_{i}{ }^{\mu}$, from which the metric tensor is derived by

$$
g^{\mu \nu} \stackrel{\text { def. }}{=} e_{i}^{\mu} e_{i}^{\nu}
$$

We assume an imaginary values for the vector $e_{0}{ }^{\mu}$ in order to have a Lorentz signature. We note that, associated with any tetrad field $e_{i}{ }^{\mu}$ there is a metric field defined uniquely by (3), while a given metric $g^{\mu \nu}$ does not determine the tetrad field completely; for any local Lorentz transformation of the tetrads $e_{i}{ }^{\mu}$ leads to a new set of tetrads which also satisfy (3). The Lagrangian $L$ is an invariant constructed from $\gamma_{\mu \nu \rho}$ and $g^{\mu \nu}$, where $\gamma_{\mu \nu \rho}$ is the contorsion tensor given by

$$
\gamma_{\mu \nu \rho} \stackrel{\text { def. }}{=} e_{i \mu} e_{i \nu ; \rho}
$$

where the semicolon denotes covariant differentiation with respect to Christoffel symbols. The most general Lagrangian density invariant under the parity operation is given by the form

$$
\mathcal{L} \stackrel{\text { def. }}{=} \sqrt{-g}\left(\alpha_{1} \Phi^{\mu} \Phi_{\mu}+\alpha_{2} \gamma^{\mu \nu \rho} \gamma_{\mu \nu \rho}+\alpha_{3} \gamma^{\mu \nu \rho} \gamma_{\rho \nu \mu}\right)
$$

where

$$
g \stackrel{\text { def. }}{=} \operatorname{det}\left(g_{\mu \nu}\right)
$$


and $\Phi_{\mu}$ is the basic vector field defined by

$$
\Phi_{\mu} \stackrel{\text { def. }}{=} \gamma_{\mu \rho}^{\rho}
$$

Here $\alpha_{1}, \alpha_{2}$, and $\alpha_{3}$ are constants determined by Møller such that the theory coincides with general relativity in the weak fields:

$$
\alpha_{1}=-\frac{1}{\kappa}, \quad \alpha_{2}=\frac{\lambda}{\kappa}, \quad \alpha_{3}=\frac{1}{\kappa}(1-2 \lambda),
$$

where $\kappa$ is the Einstein constant and $\lambda$ is a free dimensionless parameter $\theta$. The same choice of the parameters was also obtained by Hayashi and Nakano [16].

Møller applied the action principle to the Lagrangian density (5) and obtained the field equation in the form

$$
\begin{gathered}
G_{\mu \nu}+H_{\mu \nu}=-\kappa T_{\mu \nu}, \\
F_{\mu \nu}=0
\end{gathered}
$$

where the Einstein tensor $G_{\mu \nu}$ is defined by

$$
G_{\mu \nu}=R_{\mu \nu}-\frac{1}{2} g_{\mu \nu} R
$$

Here $H_{\mu \nu}$ and $F_{\mu \nu}$ are given by

$$
H_{\mu \nu} \stackrel{\text { def. }}{=} \lambda\left[\gamma_{\rho \sigma \mu} \gamma_{\nu}^{\rho \sigma}+\gamma_{\rho \sigma \mu} \gamma_{\nu}^{\rho \sigma}+\gamma_{\rho \sigma \nu} \gamma_{\mu}^{\rho \sigma}+g_{\mu \nu}\left(\gamma_{\rho \sigma \lambda} \gamma^{\lambda \sigma \rho}-\frac{1}{2} \gamma_{\rho \sigma \lambda} \gamma^{\rho \sigma \lambda}\right)\right]
$$

and

$$
F_{\mu \nu} \stackrel{\text { def. }}{=} \lambda\left[\Phi_{\mu, \nu}-\Phi_{\nu, \mu}-\Phi_{\rho}\left(\gamma_{\mu \nu}^{\rho}-\gamma_{\nu \mu}^{\rho}\right)+\gamma_{\mu \nu}{ }^{\rho} ; \rho\right]
$$

and they are symmetric and skew symmetric tensors, respectively.

Møller assumed that the energy-momentum tensor of matter fields is symmetric. In the Hayashi-Nakano theory, however, the energy-momentum tensor of spin- $1 / 2$ fundamental particles has non-vanishing antisymmetric part arising from the effects due to intrinsic spin, and the right-hand side of (10) does not vanish when we take into account the possible effects of intrinsic spin.

It can be shown 17 that the tensors, $H_{\mu \nu}$ and $F_{\mu \nu}$, consist of only those terms which are linear or quadratic in the axial-vector part of the torsion tensor, $a_{\mu}$, defined by

$$
a_{\mu} \stackrel{\text { def. }}{=} \frac{1}{3} \epsilon_{\mu \nu \rho \sigma} \gamma^{\nu \rho \sigma}
$$

where $\epsilon_{\mu \nu \rho \sigma}$ is defined by

$$
\epsilon_{\mu \nu \rho \sigma} \stackrel{\text { def. }}{=} \sqrt{-g} \delta_{\mu \nu \rho \sigma},
$$

where $\delta_{\mu \nu \rho \sigma}$ being completely antisymmetric and normalized as $\delta_{0123}=-1$. Therefore, both $H_{\mu \nu}$ and $F_{\mu \nu}$ vanish if the $a_{\mu}$ is vanishing. In other words, when the $a_{\mu}$ is found to vanish from the antisymmetric part of the field equations, (10), the symmetric part (9) coincides with the Einstein equation.

${ }^{*}$ Throughout this paper we use the relativistic units, $c=G=1$ and $\kappa=8 \pi$. 


\section{Spherically symmetric solutions}

In this section we find the most general, spherically symmetric non singular black hole solution of the form (1) in Møller's theory. The axial-vector part of the torsion tensor, $a^{\mu}$, is vanishing, and the skew part of the field equation is satisfied identically as is explained above. We discuss two cases separately: One with $S=0$ and the other with $S \neq 0$.

(i) The case without the $S$-term. In this case the axial-vector part of the torsion tensor $a^{\mu}$ is identically vanishing. Thus, when this tetrad is applied to the field equations, the skew part (10) is automatically satisfied and the solution of the symmetric part is the nonsingular black hole solution given before by Dymnikova [1]. Therefore, the solution of the form (1) with $S=0$ can be obtained from the diagonal tetrad of the nonsingular black hole metric by a local Lorentz transformation which keeps spherical symmetry,

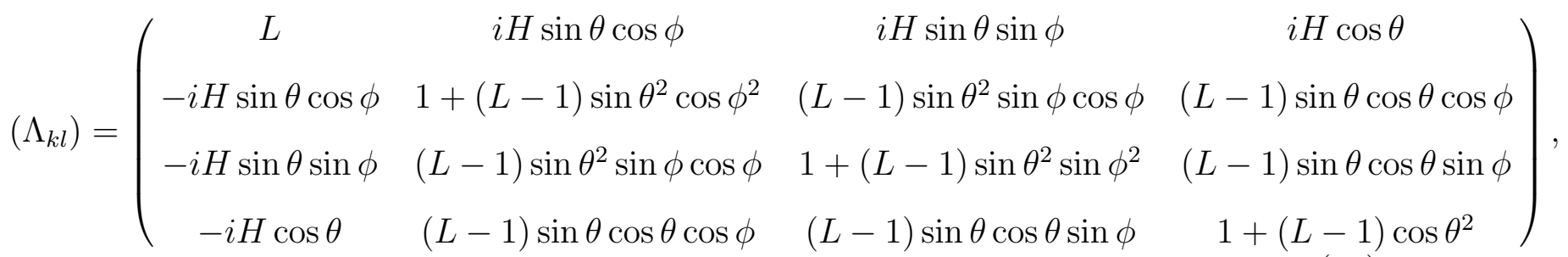

where $H$ is an arbitrary function of $t$ and $\hat{R}$ and

$$
L=\sqrt{H^{2}+1}
$$

Namely, we see that

$$
e_{i}^{\mu}=\Lambda_{i l} e_{l}^{(0) \mu}
$$

is the most general, spherically symmetric solution without the $S$-term. Here $e_{l}^{(0) \mu}$ is the diagonal tetrad in the spherical polar coordinates given by [18]

$$
\left(e_{l}^{(0) \mu}\right)=\left(\begin{array}{cccc}
\frac{i}{\hat{X}} & 0 & 0 & 0 \\
0 & \hat{X} \sin \theta \cos \phi & \frac{\cos \theta \cos \phi}{\hat{R}} & -\frac{\sin \phi}{\hat{R} \sin \theta} \\
0 & \hat{X} \sin \theta \sin \phi & \frac{\cos \theta \sin \phi}{\hat{R}} & \frac{\cos \phi}{\hat{R} \sin \theta} \\
0 & \hat{X} \cos \theta & -\frac{\sin \theta}{\hat{R}} & 0
\end{array}\right),
$$

where

$$
\hat{X}=\sqrt{1-\frac{2 m}{\hat{R}}\left(1-e^{-\hat{R}^{3} / r_{1}{ }^{3}}\right)}
$$

and $\hat{R}$ is defined as $\hat{R}=r / B$ [18]. The explicit form of the $e_{i}{ }^{\mu}$ is then given by 


$$
\left(e_{i}{ }^{\mu}\right)=\left(\begin{array}{cccc}
\frac{i L}{\hat{X}} & i H \hat{X} & 0 & 0 \\
\frac{H \sin \theta \cos \phi}{\hat{X}} & L \hat{X} \sin \theta \cos \phi & \frac{\cos \theta \cos \phi}{\hat{R}} & -\frac{\sin \phi}{\hat{R} \sin \theta} \\
\frac{H \sin \theta \sin \phi}{\hat{X}} & L \hat{X} \sin \theta \sin \phi & \frac{\cos \theta \sin \phi}{\hat{R}} & \frac{\cos \phi}{\hat{R} \sin \theta} \\
\frac{H \cos \theta}{\hat{X}} & L \hat{X} \cos \theta & -\frac{\sin \theta}{\hat{R}} & 0
\end{array}\right),
$$

where $r_{1}$ is defined by

$$
\begin{aligned}
r_{1}^{3} & =r_{g} r_{0}^{2}, \\
r_{g} & =2 m, \\
r_{0}{ }^{2} & =\frac{3}{8 \pi \epsilon_{0}} .
\end{aligned}
$$

If we apply the tetrad (19) to the symmetric part of the field equation (9), the right hand side takes the form

$$
\begin{aligned}
& T_{0}{ }^{0}=T_{1}{ }^{1}=\epsilon_{0} e^{-\hat{R}^{3} / r_{1}{ }^{3}}, \\
& T_{2}{ }^{2}=T_{3}{ }^{3}=\epsilon_{0} e^{-\hat{R}^{3} / r_{1}{ }^{3}}\left(1-\frac{3 \hat{R}^{3}}{2 r_{1}{ }^{3}}\right) .
\end{aligned}
$$

The metric associated with the tetrad (19) is given by

$$
d s^{2}=-\xi d t^{2}+\frac{d \hat{R}^{2}}{\xi}+\hat{R}^{2} d \Omega^{2},
$$

where

$$
\xi=\hat{X}^{2}, \quad \text { and } \quad d \Omega^{2}=d \theta^{2}+\sin ^{2} \theta d \phi^{2},
$$

which is the spherically symmetric nonsingular black hole solution [11.

(ii) The case with non-vanishing $S$-term. We start with the tetrad of (1) with the six unknown functions of $t$ and $r$. In order to study the condition that the $a^{\mu}$ vanishes it is convenient to start from the general expression for the covariant components of the tetrad,

$$
\begin{aligned}
e_{00} & =i \check{A}, \quad e_{a 0}=\check{C} x^{a}, \quad e_{0 \alpha}=i \check{D} x^{\alpha} \\
e_{a \alpha} & =\delta_{a \alpha} \check{B}+\check{F} x^{a} x^{\alpha}+\epsilon_{a \alpha \beta} \check{S} x^{\beta},
\end{aligned}
$$

where the six unknown functions, $\check{A}, \check{C}, \check{D}, \check{B}, \breve{F}, \breve{S}$, are connected with the six unknown functions of (1). We can assume without loss of generality that the two functions, $\check{D}$ and $\check{F}$, are vanishing by making use of the freedom to redefine $t$ and $r$ [17]. We then transform the tetrad (24) to the spherical polar coordinates $(r, \theta, \phi, t)$ to take the form

$\left(e_{i \mu}\right)=\left(\begin{array}{cccc}i A & 0 & 0 & 0 \\ r \check{C} \sin \theta \cos \phi & \check{B} \sin \theta \cos \phi & r \check{B} \cos \theta \cos \phi+r^{2} \check{S} \sin \phi & -r \check{B} \sin \theta \sin \phi+r^{2} \check{S} \sin \theta \cos \theta \cos \phi \\ r \check{C} \sin \theta \sin \phi & \check{B} \sin \theta \sin \phi & r \check{B} \cos \theta \sin \phi-r^{2} \check{S} \cos \phi & r \check{B} \sin \theta \cos \phi+r^{2} \check{S} \sin \theta \cos \theta \sin \phi \\ r \check{C} \cos \theta & \check{B} \cos \theta & -r \check{B} \sin \theta & -r^{2} \check{S} \sin ^{2} \theta\end{array}\right)$. 
Then the condition that the axial vector part $a^{\mu}$ vanishes is to be [8].

$$
0=\sqrt{(-g)} a^{\mu}= \begin{cases}3 \check{B} \check{S}+r\left(\check{B} \check{S}^{\prime}-\check{\check{B}^{\prime}} \check{S}\right), & \mu=0, \\ 2 \check{C} \check{S}+(\check{S} \check{B}-\check{S} \check{B}), & \mu=1\end{cases}
$$

with $\check{S}^{\prime}=d \check{S} / d r$ and $\check{\dot{S}}=d \check{S} / d t$. This condition can be solved to give

$$
\check{C}=0, \quad \check{S}=\frac{\eta}{r^{3}} \check{B}
$$

where $\eta$ is a constant with dimension of $(\text { length })^{2}$.

The symmetric part of the field equations now coincides with the Einstein equation. The metric tensor formed of the tetrad (25) with (27) is not of the non singular black hole solution form. Taking the new radial coordinate

$$
R=r \check{B} \sqrt{1+\frac{\eta^{2}}{r^{4}}}
$$

then the metric tensor takes the well-known non singular black hole solution form [11].

Applying the transformation (28) to the tetrad (25) with (27), we finally obtain the general, spherically symmetric non singular black hole solution with non-vanishing $S$-term: The non-vanishing, covariant components of the tetrad are given by

$$
\begin{aligned}
e_{00} & =i X \\
e_{11} & =\frac{\sin \theta \cos \phi}{X} \\
e_{12} & =\frac{R}{Y}\left(4 \eta f^{2}(R) \sin \phi+4 f^{4}(R) \cos \theta \cos \phi-\eta^{2} \cos \theta \cos \phi\right) \\
e_{13} & =\frac{R}{Y}\left(4 \eta f^{2}(R) \cos \theta \cos \phi-4 f^{4}(R) \sin \phi+\eta^{2} \sin \phi\right) \sin \theta \\
e_{21} & =\frac{\sin \theta \sin \phi}{X} \\
e_{22} & =\frac{-R}{Y}\left(4 \eta f^{2}(R) \cos \phi-4 f^{4}(R) \cos \theta \sin \phi+\eta^{2} \cos \theta \sin \phi\right) \\
e_{23} & =\frac{R}{Y}\left(4 \eta f^{2}(R) \cos \theta \sin \phi+4 f^{4}(R) \cos \phi-\eta^{2} \cos \phi\right) \sin \theta \\
e_{31}= & \frac{\cos \theta}{X} \\
e_{32}= & \frac{-R}{Y}\left(4 f^{4}(R)-\eta^{2}\right) \sin \theta \\
e_{33}= & \frac{-R}{Y} 4 \eta f^{2}(R) \sin ^{2} \theta
\end{aligned}
$$

where

$$
X=\sqrt{1-\frac{2 m}{R}\left(1-e^{-R^{3} / r^{3}}\right)}, \quad Y=4 f^{4}(R)+\eta^{2}
$$

and $f(R)$ is given by

$$
f(R)=\mathrm{e}^{\int \mathrm{dR} /(\mathrm{RX})}
$$


The tetrad (29) when applies to the symmetric part of field equation (9), the right hand side takes the form

$$
\begin{aligned}
& T_{0}^{0}=T_{1}{ }^{1}=\epsilon_{0} e^{-R^{3} / r_{1}{ }^{3}}, \\
& T_{2}{ }^{2}=T_{3}^{3}=\epsilon_{0} e^{-R^{3} / r_{1}{ }^{3}}\left(1-\frac{3 R^{3}}{2 r_{1}^{3}}\right) .
\end{aligned}
$$

The metric associated with the tetrad (29) is given by

$$
d s^{2}=-\xi_{1} d t^{2}+\frac{d R^{2}}{\xi_{1}}+R^{2} d \Omega^{2},
$$

where

$$
\xi_{1}=X^{2},
$$

which is the spherically symmetric nonsingular black hole solution [11].

It is clear that if $\eta$ and $H(\hat{R}, t)$ are equal to zero the two classes of solutions given by (19) and (29) coincide with each other, and reduce to the solution given before [18]. Furthermore, if the exponential term is equal to zero then the two solutions coincides with each other and give the Schwarzschild solution.

The solutions (19) and (29) are the exact solutions of the Møller's field equations. They practically coincide with the Schwarzschild solution for $R>>r_{1}$ or $\hat{R}>>r_{1}$ and behave like the de Sitter solution, for $R<<r_{1}$ or $\hat{R}<<r_{1}$.

As is clear from (21), the spherically symmetric stress-energy momentum tensor is really anisotropic. The difference between the principle pressures

$$
T_{k}^{k}=-p_{k},
$$

correspond to the well known anisotropic character of evolution of the space-time inside a black hole undergoing a spherically symmetric gravitational collapse [19]. For $R<<r_{1}$ isotropization occurs and the stress-energy momentum tensor takes the isotropic form

$$
T_{\alpha \beta}=\epsilon g_{\alpha \beta} .
$$

When $R \rightarrow 0$ the energy density tends to $\epsilon_{0}$. For $R>>r_{1}$ all the components of the stressenergy momentum tensor tend to zero very rapidly. The same properties satisfied for the stress-energy momentum tensor (31).

Now let us compare the solution (19) with that given before [15]. Started from a spherically symmetric tetrad with three unknown functions of the radial coordinate $r$ only, which is given in the spherical polar coordinates by

$$
\left(e_{i}{ }^{\mu}\right)=\left(\begin{array}{cccc}
i A & i D r & 0 & 0 \\
0 & B \sin \theta \cos \phi & \frac{B}{r} \cos \theta \cos \phi & -\frac{B \sin \phi}{r \sin \theta} \\
0 & B \sin \theta \sin \phi & \frac{B}{r} \cos \theta \sin \phi & \frac{B \cos \phi}{r \sin \theta} \\
0 & B \cos \theta & -\frac{B}{r} \sin \theta & 0
\end{array}\right) .
$$


Applying the tetrad (36) to the field equations, (9) and (10), we obtained a general solution of the form

$$
\begin{aligned}
& \mathcal{A}(\hat{R})=\frac{1}{1-\hat{R} \mathcal{B}^{\prime}}, \\
& \mathcal{D}(\hat{R})=\frac{1}{1-\hat{R} \mathcal{B}^{\prime}} \sqrt{\frac{2 m}{\hat{R}^{3}}\left(1-\mathrm{e}^{\left(-\hat{\mathrm{R}}^{3} / \mathrm{r}_{1}{ }^{3}\right)}\right)+\frac{\mathcal{B}^{\prime}}{\hat{R}}\left(\hat{R} \mathcal{B}^{\prime}-2\right)},
\end{aligned}
$$

where $\mathcal{A}(\hat{R}), \quad \mathcal{B}(\hat{R}) \quad$ and $\quad \mathcal{D}(\hat{R})$ are connected to $A, B$ and $D$ through the transformation $\hat{R}=r / B, \quad \mathcal{B}^{\prime}=\frac{d \mathcal{B}(\hat{R})}{d \hat{R}}, \quad$ and $\mathcal{B}$ is an arbitrary function of $\hat{R}$. The tetrad (36) can further be expressed in the form [15]

$$
\left(e_{i}{ }^{\mu}\right)=\left(\begin{array}{cccc}
\frac{i \mathcal{A}}{1-\mathcal{D}^{2} \hat{R}^{2}} & i \mathcal{D} \hat{R}\left(1-\hat{R} \mathcal{B}^{\prime}\right) & 0 & 0 \\
\frac{\mathcal{A D} \hat{R} \sin \theta \cos \phi}{1-\mathcal{D}^{2} \hat{R}^{2}} & \left(1-\hat{R} \mathcal{B}^{\prime}\right) \sin \theta \cos \phi & \frac{\cos \theta \cos \phi}{\hat{R}} & -\frac{\sin \phi}{\hat{R} \sin \theta} \\
\frac{\mathcal{A D} \hat{R} \sin \theta \sin \phi}{1-\mathcal{D}^{2} \hat{R}^{2}} & \left(1-\hat{R} \mathcal{B}^{\prime}\right) \sin \theta \sin \phi & \frac{\cos \theta \sin \phi}{\hat{R}} & \frac{\cos \phi}{\hat{R} \sin \theta} \\
\frac{\mathcal{A D} \hat{R} \cos \theta}{1-\mathcal{D}^{2} \hat{R}^{2}} & \left(1-\hat{R} \mathcal{B}^{\prime}\right) \cos \theta & \frac{-\sin \theta}{\hat{R}} & 0
\end{array}\right) .
$$

Thus, it is easy to verify that this tetrad can be obtained from (19) by choosing the function $H$ as

$$
H=\frac{\left[\hat{R}^{2} \mathcal{B}^{\prime 2}-2 \hat{R} \mathcal{B}^{\prime}+\frac{2 m}{\hat{R}}\left(1-\mathrm{e}^{\left(-\hat{\mathrm{R}}^{3} / \mathrm{r}_{1}{ }^{3}\right)}\right)\right]^{1 / 2}}{\sqrt{1-\frac{2 m}{\hat{R}}\left(1-e^{-\hat{R}^{3} / r_{1}{ }^{3}}\right)}}
$$

\section{The Energy Associated with each Solution}

The superpotential of the Møller's tetrad theory of gravitation is given by Mikhail et al. [7] as

$$
\mathcal{U}_{\mu}{ }^{\nu \lambda}=\frac{(-g)^{1 / 2}}{2 \kappa} P_{\chi \rho \sigma}{ }^{\tau \nu \lambda}\left[\Phi^{\rho} g^{\sigma \chi} g_{\mu \tau}-\lambda g_{\tau \mu} \gamma^{\chi \rho \sigma}-(1-2 \lambda) g_{\tau \mu} \gamma^{\sigma \rho \chi}\right]
$$

where $P_{\chi \rho \sigma}{ }^{\tau \nu \lambda}$ is

$$
P_{\chi \rho \sigma}{ }^{\tau \nu \lambda} \stackrel{\text { def. }}{=} \delta_{\chi}{ }^{\tau} g_{\rho \sigma}{ }^{\nu \lambda}+\delta_{\rho}^{\tau} g_{\sigma \chi}{ }^{\nu \lambda}-\delta_{\sigma}{ }^{\tau} g_{\chi \rho}{ }^{\nu \lambda}
$$

with $g_{\rho \sigma}{ }^{\nu \lambda}$ being a tensor defined by

$$
g_{\rho \sigma} \nu \lambda \stackrel{\text { def. }}{=} \delta_{\rho}{ }^{\nu} \delta_{\sigma}^{\lambda}-\delta_{\sigma}{ }^{\nu} \delta_{\rho}^{\lambda}
$$


The energy is expressed by the surface integral [20]

$$
E=\lim _{r \rightarrow \infty} \int_{r=\text { constant }} \mathcal{U}_{0}^{0 \alpha} n_{\alpha} d S
$$

where $n_{\alpha}$ is the unit 3 -vector normal to the surface element $d S$.

Now we are in a position to calculate the energy associated with the two solutions (19) and (29) using the superpotential (40). As is clear from (43), the only component which contributes to the energy is $\mathcal{U}_{0}{ }^{0 \alpha}$. Thus substituting from the solution (19) into (40) we obtain the following non-vanishing value

$$
\mathcal{U}_{0}^{0 \alpha}=\frac{2 \hat{X} x^{\alpha}}{\kappa \hat{R}}(L-\hat{X}) .
$$

Substituting from (44) into (43) we get

$$
E(\hat{R})=\hat{X} \hat{R}(L-\hat{X})
$$

As is clear from (45) that the energy depends on the arbitrary function $H$. If this arbitrary function takes the value (39) then

$$
E(\hat{R})=2 m\left(1-e^{-\hat{R}^{3} / r_{1}{ }^{3}}\right)-\hat{R}^{2} \mathcal{B}^{\prime}
$$

which is the same as that obtained before [15].

Now let us turn our attention to the solution (29). Calculating the necessary components of the superpotential, we get

$$
\mathcal{U}_{0}^{0 \alpha}=\frac{4 X x^{\alpha}}{\kappa R}\left(\frac{\left(4 f(R)^{4}-\eta^{2}\right)}{Y}-X\right) .
$$

Substituting from (47) into (43) we get

$$
E(R)=X R\left(\frac{\left(4 f(R)^{4}-\eta^{2}\right)}{Y}-X\right)
$$

where $f(R)$ is given by (30). As is clear from (48) that if the constant $\eta=0$, then

$$
E(R)=m\left(1-e^{-R^{3} / r_{1}^{3}}\right),
$$

which is the same as that given before [18], Yang [21] and Radinschi [22]. This is a very satisfactory result since the two tetrads (29), (36) coincide when $\eta=0$ and $D=0$, and accordingly their energies must be identical. We can also see from (48) that if $R \rightarrow 0$, then $E(R) \rightarrow 0$ and form (49) if $R \rightarrow \infty$ then $E(R) \rightarrow m$. Thus the total energy will coincide with that of the Schwarzschild solution. 


\section{Main results and Discussion}

We have studied the general spherically symmetric nonsingular black hole solutions in teleparallel theory of gravitation. Tetrads with spherical symmetry are classified into two groups.

i) The groups in which the S-term is vanishing, and therefore, the axial vector part $a^{\mu}$ is identically vanishing. Accordingly we obtain a family of solutions with arbitrary function of t and $\hat{R}$, i.e., $H(t, \hat{R})$. A relation with the solution given before 15 had been established by assuming a specific form of the arbitrary function $H(t, \hat{R})$ as given by (39).

ii) The groups which possess the S-term, namely, the term $S(t, r) \epsilon_{a \alpha \beta} x^{\beta}$. When the S-term is non-vanishing, we obtain the condition (27) which is the condition for vanishing the axial vector part, i.e. $a^{\mu}=0$. In this case we obtain a family of solutions with a constant parameter, i.e., $\eta$. For comparison if this constant is set equal to zero then the tetrad (29) will reduce to the diagonal form of (36).

Pereira et al. [23] studied the teleparallel version of the exact solutions ( Schwarzschild and Kerr solutions) of general relativity. As is well known that the torsion tensor can be decomposed into three parts tracless part, vector part and the axial vector part [17]. Using the weak-field limit they [23] show that the vector and the tracless parts of the Schwarzschild torsion combine to yield the Newtonian force. By considering the slow rotation and weakfield approximation they show that for the Kerr solution the axial vector part is nothing but the gravitomagnetic components of the gravitational field and is therefore the responsible for the Lense Thirring effect.

We also calculated the energy associated with the two solutions (19) and (29). Concerning the energy associated with the solution without the S-term, it is found that the energy depends on the arbitrary function $H(t, \hat{R})$. If the function $H(t, \hat{R})$ takes the value (39), then the energy coincides with that obtained before 15] and if $H(t, R)=0$, then the energy coincides with that obtained by Yang [21] and Radinschi 222]

As for the energy associated with the solution when the S-term is non-vanishing we obtain the expression (48). It is clear from this expression that the energy depends on the parameter $\eta$ and if this parameter equals zero then the tetrad (29) will coincide with the diagonal form of the tetrad (36) and the associated energy will be the same as that obtained before [18]. It is clear from (48), that if $R \rightarrow 0$, then $E(R) \rightarrow 0$ and from (49) if $R \rightarrow \infty$ then $E(R) \rightarrow m$. Thus the total energy will coincide with that of the Schwarzschild solution, since the metric associated with the tetrad (29) in this case gives the Schwarzschild metric.

It is readily seen from equations (46) and (49) that the factors in front of the gravitational mass $\mathrm{m}$ are different. This is because that when $H(t, \hat{R})=0$, the components $\left(e_{a 0}\right)$ and $\left(e_{0 \alpha}\right)$ of the parallel vector fields (19) tend to zero as $1 / \sqrt{\hat{R}}$ and in that case the gravitational mass will not coincide with the inertial mass even in the simple case of vacuum, i.e., $\epsilon_{0}=0$ [7, 8, 24]. In contrast to the tetrad (29) when $\eta=0$, the components $\left(e_{a 0}\right)$ and $\left(e_{0 \alpha}\right)$ tend to zero faster than $1 / \sqrt{R}$ and this case is satisfactory since the gravitational mass equals to the inertial mass [7, 8].

In the case of the ADM mass we obtain the same result as we can see from (49)

$$
M_{A D M}=E(R)_{R \rightarrow \infty}=m .
$$

But for equation (46) the result will depend on the form of the arbitrary function $\mathcal{B}$.

We calculate the energy using expression (43) mainly given by Møller [1]. Blagojevie $e^{\prime}$ and Vasilie $^{\prime}$ 25] presented an investigation of the connection between the asymptotic Poincare $e^{\prime}$ 
symmetry of space-time and the related conservation laws of energy-momentum and angular momentum in teleparallel theory of gravity. They [25] derived the generators of the global Poincare $e^{\prime}$ symmetry in the asymptotic region from the related gauge generators. They [25] concluded that the Poincare $e^{\prime}$ generators have to be improved by adding certain surface terms, which represent the values of energy-momentum and angular momentum of the gravitating system. These results for energy-momentum and angular momentum are valid for general teleparallel theory of gravity.

Chang et al. 26 argued that every energy-momentum complex is associated with a legitimate Hamiltonian boundary term, and, because of this the energy-momentum complexes are quasi-local and acceptable. Each is the energy-momentum density for some physical situation. This Hamiltonian approach to quasi-local energy-momentum rehabilitates of the energy-momentum complexes.

A summary of the main results is given in the table below. The general solution of spherically symmetric nonsingular black hole is classified into two groups according to whether or not the space-space components $e_{a}{ }^{\alpha}$ have the term $S \epsilon_{a \alpha \beta} x^{\beta}$ (referred to as the $S$-term for short). The general solution without the $S$-term has an arbitrary function of $t$ and $r$. The general solution with the $S$-term has a constant parameter.

\begin{tabular}{|c|c|c|c|}
\hline & \multicolumn{2}{|c|}{ Field equation } & Energy \\
\cline { 2 - 3 } $\begin{array}{c}\text { skew part } \\
\text { Tetrad }\end{array}$ & Symmetric part & \\
\hline without the $S$-term & $\begin{array}{c}\text { Satisfied } \\
\text { identically }\end{array}$ & $\begin{array}{c}\text { Nonsingular black } \\
\text { hole solution }\end{array}$ & $\hat{X}(L-\hat{X})$ \\
\hline Tetrad & Gives & Nonsingular black & $X R\left(\frac{\left(4 f(R)^{4}-\eta^{2}\right)}{Y}-X\right)$ \\
with $S$-term & $a^{\mu}=0$ & hole solution & \\
\hline
\end{tabular}

\section{Acknowledgements}

The author would like to thank Professor I.F.I. Mikhail; Ain Shams University, for his stimulating discussions. 


\section{References}

[1] C. Møller, Mat. Fys. Medd. Dan. Vid. Selsk. 39 (1978), 13.

[2] C. Møller, Mat. Fys. Medd. Dan. Vid. Selsk. 1 (1961), 10.

[3] D. Sáez, Phys. Rev. D27 (1983), 2839.

[4] H. Meyer, Gen. Rel. Grav. 14 (1982), 531.

[5] K. Hayashi and T. Shirafuji, Prog. Theor. Phys. 64, 866, 883, 1435, 2222; 65 (1980), 525

[6] F.W. Hehl, J. Nitsch and P. von der Heyde, in General Relativity and Gravitation, A. Held, ed. (Plenum Press, New York) (1980).

[7] F.I. Mikhail, M.I. Wanas, A. Hindawi and E.I. Lashin, Int. J. of Theor. Phys. 32(1993), 1627.

[8] T. Shirafuji, G.G.L. Nashed and K. Hayashi, Prog. Theor. Phys. 95 (1996), 665.

[9] F.I. Mikhail, M.I. Wanas, I.E. Lashin and A. Hindawi Gen. Rel. Grav. 26 (1994), 869.

[10] A. Mazumder and D. Ray, Internat. J. Theor. Phys. 29 (1990), 431.

[11] I.G. Dymnikova, Gen. Rel. Grav. 24 (1992), 235.

[12] I.G. Dymnikova, Phys. Lett. B472 (2000), 33.

[13] I.G. Dymnikova, A. Dobosz, M.L. Fil'chenkov and A. Gromov Phys. Lett. B 506 (2001), 351.

[14] H.P. Robertson, Ann. of Math. (Princeton) 33 (1932), 496.

[15] G.G.L. Nashed, Gen. Rel. Grav. 34 (2002), 1047.

[16] K. Hayashi and T. Nakano, Prog. Theor. Phys. 38 (1967), 491.

[17] K. Hayashi and T. Shirafuji, Phys. Rev. D19 (1979), 3524.

[18] G.G.L. Nashed, Accepted for publication in IL Nuovo Cimento B ; gr-qc/0109017

[19] Ya B. Zeldovich and I.D. Novikov, The Structure and Evolution of the Universe (Chicago Univ. Press, Chicago) (1983).

[20] C. Møller, Ann. of Phys. 4 (1958), 347.

[21] I -C Yang, Chin. J. Phys. 38 (2000), 1040.

[22] I. Radinschi, Mod. Phys. Lett. A15 (2000), 803.

[23] J.G. Pereira, T. Vargas and C. M. Zhang, Class. Quant. Grav. 18 2001, 833.

[24] T. Shirafuji, G.G.L. Nashed and Y. Kobayashi, Prog. Theor. Phys. 96 (1996), 933.

[25] M. Blagojevie' and M Vasilie', Phys. Rev. D64 (2001), 044010.

[26] Chia-Chen Chang, J.M. Nester and Chiang-Mei Chen, Phys. Rev. Lett. 83 (1999), 1897; gr-qc/9912058. 